\title{
L'INTERVENTION D'UNE HORMONE, LA SURVIVONE, DANS LA MORTALITÉ DES ABEILLES (APIS MELLIFICA L.) ISOLÉES (EFFET DE GROUPE)
}

\author{
Rémy CHAUVIN *, Jean-Pierre LAFARGE ** et Jean-Pierre SALIGOT ** \\ * Laboratoire de Sociologie animale, Université René-Desartes, \\ Le Château, 18380 Ivoy-le-Pré \\ ** Laboratoire antidopage, Ministère de la Jeunesse et des Sports \\ 106, quai de Clichy, 92110 Clichy
}

\begin{abstract}
RESUME
L'acide azélaïque et l'acide pimélique présents dans les extraits d'abeilles ouvrières prolongent notablement la vie de l'abeille isolée. Ils agissent en réduisant les échanges gazeux de ces abeilles.
\end{abstract}

\section{INTRODUCTION}

L'effet de groupe consiste chez les abeilles en une mortalité des abeilles isolées bien plus grande que celle des groupées (Grassé et Chauvin, 1944). Ce phénomène dépend de la saison et il disparaît pratiquement en mai; il dépend aussi du poids car il existe de grandes différences de ce point de vue entre les ouvrières, qui proviennent des conditions d'alimentation des larves qui leur ont donné naissance. L'effet de groupe apparaît dès que deux abeilles sont réunies, même si la seconde abeille est morte : on verra plus loin l'importance de cette observation (ARNOLD, 1976). Du point de vue physiologique l'isolement augmente fortement le métabolisme respiratoire (RоTH, 1965); il n'agit ni sur les glandes hypopharyngiennes ni sur les cirières, ni sur la teneur en eau et en sucres (SitBon, 1971 ; ARNOLD, 1976) ; mais les lipides sont à un taux moins élevé chez l'isolée ; les ovaires des isolées ne se développent pas et la pars intercerebralis présente moins de sécrétion que celle des groupées (Sitbon,1971; ArNold, 1976).

L'observation d'ARNold suivant laquelle la présence d'une abeille morte auprès d'une abeille vivante induit une survie analogue à celle d'une autre abeille 
vivante évoque très nettement une action chimique et la présence probable d'une phéromone; on peut rappeler à cette occasion les travaux de PAIN (1961) qui découvrit qu'un cadavre de reine inhibait aussi bien le développement de l'ovaire des ouvrières qu'une reine vivante, ce qui l'amena à isoler, avec BARBIER (PAin et Barbier, 1963), la première phérormone. Mais les premiers essais d'extraction de la phérormone hypothétique de l'ouvrière montrèrent surtout la toxicité des extraits d'abeilles par l'alcool bouillant (Chauvin, 1979). La seule méthode adéquate consiste dans une macération des cadavres frais dans l'éther éthylique pendant trois heures, suivie d'une précipitation par l'alcool. Le précipité, encore très complexe, est fort actif : je lui ai donné le nom de «survivone» (Chauvin, 1981). Avec Lafarge et Saligot, nous sommes parvenus à purifier la survivone et à en isoler les facteurs actifs.

TABL. 1. - Essai du distillat sur la mortalité des abeilles isolées.

TABL. 1. - Action of distillated part upon isolated bees mortality.

\begin{tabular}{|c|c|c|c|}
\hline \multirow{2}{*}{$\begin{array}{l}\text { Date } \\
\text { Date }\end{array}$} & \multirow{2}{*}{$\begin{array}{l}\text { Nombre d'abeilles } \\
\text { testées } \\
\text { Number of honeybees } \\
\text { tested }\end{array}$} & \multicolumn{2}{|c|}{$\begin{array}{l}\text { Nombre d'abeilles mortes au bout de } \\
70-75 \text { heures } \\
\text { Number of dead honeybees after } 70-75 \text { hours }\end{array}$} \\
\hline & & $\begin{array}{l}\text { Témoin } \\
\text { Control }\end{array}$ & $\begin{array}{c}\text { Traitées } \\
\text { Treated bees }\end{array}$ \\
\hline 21-1-1981 & 40 & 18 & 7 \\
\hline $25-1-1981$ & 40 & 16 & 11 \\
\hline 2-2-1982 & 40 & 18 & 13 \\
\hline 6-2-1982 & 40 & 16 & 10 \\
\hline $9-2-1982$ & 40 & 16 & 5 \\
\hline $9-2-1982$ & 40 & 16 & 10 \\
\hline $20-2-1982$ & 40 & 16 & 14 \\
\hline $20-2-1982$ & 40 & 17 & 14 \\
\hline $20-2-1982$ & 40 & 16 & 13 \\
\hline
\end{tabular}

\section{MATERIEL ET METHODES}

\section{Purification}

On obtient une purification notable en pratiquant un entraînement par la vapeur d'eau. Quelques centaines de milligrammes de précipité sont placés dans un ballon de $50 \mathrm{~cm}^{3}$ avec $250 \mathrm{~cm}^{3}$ d'eau bouillante : la vapeur laisse déposer dans le réfrigérant des paillettes blanchâtres très actives sur la survie. Ce sont ces paillettes que LAFARgE et SALIGot ont examinées au moyen du chromatographe en phase gazeuse (système G.C.-M.S. Hewlett Packard; colonne capillaire de silice fondue de $25 \mathrm{~m}$ apolaire, injecteur à aiguilles). L'échantillon a été analysé sous sa forme initiale, après méthylation et après silylation. 


\section{Technique des tests.}

L'échantillon sur lequel portent les expériences est composé de 40 abeilles isolées traitées et 40 témoins (mais évidemment on peut faire plusieurs expériences à la fois ce qui porte souvent le nombre des abeilles en expérience à 400. L'isolement est pratiqué dans des manchons de plastique sans fond de $3 \times 4 \mathrm{~cm}$ (dimensions intérieures). Les produits à tester sont déposés à raison de $1 \mu \mathrm{g}$ par abeille sur un support dont la nature est importante : il faut qu'il soit rugueux et non pas lisse. Je me sers de tampons de coton à démaquiller de $5 \mathrm{~cm}$ de diamètre coupés en 16 portions après qu'on les a séparés en trois dans le sens de l'épaisseur. Les abeilles sont introduites sous anesthésie à l'éther dans les manchons et l'ensemble porté à $30^{\circ}$ dans une pièce de quatre mètres cubes où l'on a pu réaliser l'homogénéisation parfaite de la température pour tous les échantillons : c'est une précaution absolument essentielle.

Les abeilles ne reçoivent qu'une pastille de sucre candi. On compte la mortalité des abeilles et on s'arrête dès que $50 \%$ des témoins sont morts. Cela se produit au bout d'environ 70 heures.

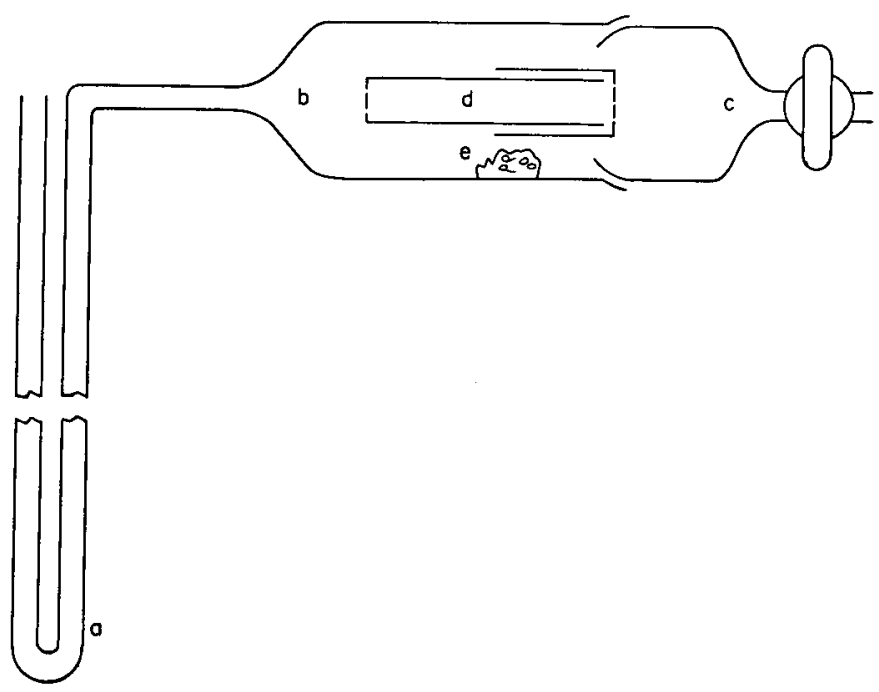

Fig. 1. - Le respiromètre. a, tube manométrique (diamètre intérieur $2 \mathrm{~mm}$, longueur du tube $35 \mathrm{~cm}$ ); b, chambre manométrique (longueur $12 \mathrm{~cm}$, diamètre intérieur $2 \mathrm{~cm}$ ); c, robinet.; d, tube qui contient l'abeille en cours d'expérience; e, tampon imbibé d'une solution de $\mathrm{NaOH}$.

FIG. 1. - The respirometer. a, manometric tube $(2 \mathrm{~mm}$ diameter length $35 \mathrm{~cm})$; b, manometric chamber (length $12 \mathrm{~cm}$ internal diameter $2 \mathrm{~cm}$ ); $c$, tap; d, tube for bee in experiment ; e, cotton pad soaked with $\mathrm{NaOH}$ solution.

Le problème consistait à mesurer en même temps le métabolisme d'une série d'abeilles témoins et d'abeilles soumises à l'action des substances énumérées plus haut. Il est difficile d'employer un appareil de Warburg car il nécessite l'agitation qui perturberait les sujets. J'ai trouvé avantage à me servir d'un «respiromètre»: ces appareils ont été beaucoup employés dans l'étude des insectes de petite dimension parce qu'on peut leur donner la sensibilité qu'on veut en réduisant le calibre du tube mesureur. Le modèle utilisé est un manomètre à alcool dont le tube manométrique est de $2 \mathrm{~mm}$ de diamètre. Les sujets sont enfermés dans un petit tube de verre dont 
les deux extrémités sont fermées par une toile métallique. On dispose à côté, dans la chambre manométrique, une boulette de coton trempée dans une solution normale de soude puis sommairement essorée; la soude se trouve en grand excès par rapport à la quantité de gaz à absorber. Après un repos d'une demi-heure, on ferme le robinet du manomètre; on opère une première lecture après 20 minutes; on ouvre le robinet pour remettre la colonne à zéro et on refait deux autres lectures. L'appareil ne fonctionne correctement que dans une pièce de petites dimensions, sans courants d'air: sinon il fonctionne très évidemment comme un thermomètre à gaz. La température à laquelle cette pièce doit être portée est de $22^{\circ}$; elle est suffisamment basse pour que les abeilles ne soient pas trop actives et suffisamment élevée pour qu'elles ne tombent pas en état de torpeur due au froid. Il va de soi que pour chaque sujet traité on réserve un témoin. On sait par ailleurs quelle est la difficulté d'établir un métabolisme de base chez un poïkilotherme. Toujours est-il qu'à condition de ne pas demander à l'appareil une précision qu'il ne peut apporter et à condition de réserver des témoins les résultats m'ont paru fort utilisables. Une des difficultés principales est toutefois la différence formidable de l'émission de gaz carbonique entre individus, et chez le même individu. Différents auteurs (PunT, 1950) ont d'ailleurs établi le caractère irrégulier de l'émission de gaz carbonique chez certains insectes où elle survient en quelque sorte par bouffées. Cela complique beaucoup les mesures et oblige à augmenter leur nombre. Une autre difficulté est la différence de poids entre abeilles : j'ai essayé de me rapprocher le plus possible du poids de 100 mgrs.

\section{RESULTATS}

On trouve dans la survivone :
a) une série de diacides de $\mathrm{C} 6$ à $\mathrm{C} 10$;
b) une série de monoacides de $\mathrm{C} 12$ à $\mathrm{C} 22$;
c) des alcools supérieurs en C 16, C 18, C 20 ;
d) la série des alcanes de C 21 à C 29.

Seuls deux diacides, le pimélique (heptane dioïque) et l'azélaïque (nonane dioïque) sont actifs ; l'acide nonanoïque est inactif. L'acide azélaïque a déjà été signalé dans la tête des abeilles ou la gelée royale (LERCKER et coll., 1981).

Un autre corps isolé par LENSKY dans les traces que laissent les pattes des abeilles sur le substrat, l'acide décanoïque, est nettement actif, mais il ne se trouve pas dans la survivone. J'ai été aussi fort étonné de n'y pas rencontrer les acides insaturés comme les composés de l'acide décanoïque qui ont été si bien étudiés à la suite des travaux de PaIN et qui sont abondants dans la gelée royale.

D'autre part, les corps actifs sur la survie réduisent tous le métabolisme et diminuent le rejet de $\mathrm{CO}_{2}$. Toutefois, il faut apporter tout de suite certaines restrictions à cette affirmation : il existe des corps (signalés par LENSKY) que j'ai trouvés inactifs sur la survie, comme le cyclopentanol et qui réduisent l'émission de gaz carbonique. Il est donc bien possible que la survivone n'agisse pas uniquement par réduction du métabolisme des isolées. 
TABL. 2. - Action des acides azélaïque, pimélique et caprique (ou décanö̈que) sur la mortalité des abeilles isolées. Les deux premiers acides sont des diacides présents dans la survivone, le troisième est un monoacide absent de la survivone mais présent dans les extraits tarsaux de LENSKY.

TABL. 2. - Influence of azelaic, pimelic and capric (or decanoic) acids on the mortality of isolated honeybees. The first two acids are diacids present in survivone, the third one is a monoacid absent in survivone but present in tarsal extracts (LeNSKY).

\begin{tabular}{|c|c|c|c|c|c|c|c|}
\hline \multirow{3}{*}{ Date } & \multirow{3}{*}{$\begin{array}{c}\text { Nombre } \\
\text { d'abeilles } \\
\text { testées } \\
\text { Number of } \\
\text { honeybees } \\
\text { tested }\end{array}$} & \multicolumn{6}{|c|}{$\begin{array}{c}\text { Nombre d'abeilles mortes au bout de } 70-75 \text { heures } \\
\text { Number of dead honeybees after } 70-75 \text { hours }\end{array}$} \\
\hline & & \multicolumn{2}{|c|}{$\begin{array}{c}\text { Acide azélaïque } \\
\text { Azelaic acid }\end{array}$} & \multicolumn{2}{|c|}{$\begin{array}{l}\text { Acide pimélique } \\
\text { Pimelic acid }\end{array}$} & \multicolumn{2}{|c|}{$\begin{array}{l}\text { Acide caprique } \\
\text { Capric acid }\end{array}$} \\
\hline & & Témoins & Traitées * & Témoins & Traitées * & Témoins & Traitées * \\
\hline \multirow[t]{4}{*}{$25-11-1983$} & 40 & 20 & 11 & & & & \\
\hline & 40 & 24 & 15 & & & & \\
\hline & 40 & 19 & 12 & & & & \\
\hline & 40 & 19 & 12 & & & & \\
\hline \multirow[t]{4}{*}{$1-12 \cdot 1983$} & 40 & 24 & 15 & & & & \\
\hline & 40 & 20 & 16 & & & & \\
\hline & 40 & 20 & 16 & & & & \\
\hline & 40 & 21 & 18 & & & & \\
\hline \multirow[t]{4}{*}{$27-4-1984$} & 40 & & & 26 & 12 & & \\
\hline & 40 & & & 11 & 19 & & \\
\hline & 40 & & & 22 & 14 & & \\
\hline & 40 & & & 15 & 14 & & \\
\hline \multirow[t]{4}{*}{$12-5-1984$} & 40 & & & 10 & 10 & & \\
\hline & 40 & & & 19 & 12 & & \\
\hline & 40 & & & 22 & 12 & & \\
\hline & 40 & & & 20 & 12 & & \\
\hline \multirow[t]{4}{*}{ 4- $9-1983$} & 40 & & & & & 17 & 14 \\
\hline & 40 & & & & & 21 & 17 \\
\hline & 40 & & & & & 17 & 13 \\
\hline & 40 & & & & & 20 & 9 \\
\hline \multirow[t]{4}{*}{ 14- $9-1983$} & 40 & & & & & 15 & 10 \\
\hline & 40 & & & & & 16 & 17 \\
\hline & 40 & & & & & 15 & 15 \\
\hline & 40 & & & & & 17 & 13 \\
\hline
\end{tabular}

* Test de Student significatif à $5 \%$.

Student test significant at $5 \%$. 
TaBL. 3. - Action de divers corps sur la dénivellation manométrique (en mm, tube manométrique de $2 \mathrm{~mm}$ de diamètre). Test de Student significatif à $1 \%$ pour les acides azélaïque et pimélique, à $5 \%$ pour le Mann-Whitney, Mann et Whitney significatif à moins de $1 \%$ pour le cyclopenthanol.

TABL. 3. - Action of several substances upon manometric denivellation (in $\mathrm{mm}$, manometric tube diameter : $2 \mathrm{~mm}$ ). Student test positive at $1 \%$ for azelaic and pimelic acids; at $5 \%$ for Mann \& Whitney test. Mann \& Whitney test positive at less thann $1 \%$ for cyclopentanol.

\begin{tabular}{|c|c|c|c|c|c|}
\hline Acide pimélique & $\mathrm{T}$ & Acide azélaïque & $\mathbf{T}$ & Cyclopentanol & $\mathrm{T}$ \\
\hline 2 & 10 & 135 & 145 & 30 & 20 \\
\hline 5 & 15 & 140 & 155 & 190 & 190 \\
\hline 15 & 15 & 40 & 190 & 70 & 195 \\
\hline 15 & 15 & 35 & 180 & 26 & 65 \\
\hline 17 & 18 & 70 & 105 & 65 & 20 \\
\hline 6 & 16 & 20 & 22 & 22 & 55 \\
\hline 6 & 22 & 25 & 80 & 19 & 35 \\
\hline 6 & 10 & 55 & 40 & 65 & 45 \\
\hline 20 & 25 & 20 & 37 & 20 & 140 \\
\hline 6 & 10 & 15 & 25 & 180 & 190 \\
\hline 15 & 6 & 26 & 34 & 175 & 195 \\
\hline 25 & 15 & 17 & 40 & 160 & 170 \\
\hline 20 & 6 & 25 & 87 & 120 & 80 \\
\hline 6 & 6 & 30 & 37 & 70 & 150 \\
\hline 6 & 10 & 15 & 37 & 60 & 110 \\
\hline 20 & 180 & 30 & 10 & 43 & 45 \\
\hline 70 & 28 & 15 & 58 & 20 & 45 \\
\hline 28 & 165 & 20 & 15 & 30 & 55 \\
\hline 45 & 165 & 45 & 15 & 30 & 30 \\
\hline 180 & 25 & 15 & 100 & 23 & 25 \\
\hline 46 & 195 & 10 & 20 & 30 & 30 \\
\hline 190 & 195 & 35 & 150 & 185 & 160 \\
\hline 40 & 140 & 65 & 45 & 190 & 160 \\
\hline 170 & 190 & 15 & 20 & 195 & 195 \\
\hline 75 & 20 & 105 & 50 & 130 & 55 \\
\hline 180 & 37 & 65 & 95 & 160 & 140 \\
\hline 25 & 25 & 25 & 19 & 125 & 135 \\
\hline 36 & 37 & 50 & 20 & 50 & 35 \\
\hline 140 & 55 & 18 & 125 & 30 & 47 \\
\hline \multirow[t]{3}{*}{45} & 30 & 28 & 180 & 48 & 54 \\
\hline & & 66 & 150 & 10 & 180 \\
\hline & & 50 & 25 & 10 & 110 \\
\hline
\end{tabular}


TABL. 3 (suite)

\begin{tabular}{|c|c|c|c|c|c|}
\hline Acide pimélique & $\mathrm{T}$ & Acide azélaïque & $\mathrm{T}$ & Cyclopentanol & $\mathrm{T}$ \\
\hline & & 47 & 18 & 50 & 110 \\
\hline & & 60 & 180 & 40 & 100 \\
\hline & & 42 & 107 & 21 & 180 \\
\hline & & 156 & 190 & 100 & 61 \\
\hline & & 130 & 112 & 127 & 52 \\
\hline & & 30 & 15 & 50 & 28 \\
\hline & & 39 & 10 & 50 & 75 \\
\hline & & 45 & 160 & 50 & 50 \\
\hline & & 30 & 20 & 26 & 25 \\
\hline & & 109 & 140 & 23 & 70 \\
\hline & & 30 & 70 & 23 & 25 \\
\hline & & 25 & 10 & 40 & 150 \\
\hline & & 30 & 90 & 25 & 15 \\
\hline & & 22 & 45 & 17 & 120 \\
\hline & & 17 & 20 & 30 & 30 \\
\hline & & 30 & 57 & 30 & 5 \\
\hline & & 20 & 37 & 20 & 190 \\
\hline
\end{tabular}

Le siège d'émission de la survivone. Le test respirométrique a été appliqué à la recherche du siège d'émission de la survivone. J'ai d'abord recherché si la présence d'un cadavre réduit l'émission de gaz carbonique chez une abeille vivante : et c'est bien le cas. Il était alors facile de présenter aux abeilles isolées soit une tête isolée, soit un corps entier dépourvu de tête : les résultats sont tout à fait nets : c'est la tête qui induit la réduction de l'émission de $\mathrm{CO}_{2}$. Il me parâtt donc probable que la survivone est sécrétée dans la tête et probablement dans les glandes mandibulaires. Toutefois, l'inactivité de l'essence de gelée royale totale préparée comme la survivone par entraînement à la vapeur d'eau, montre, ou bien qu'il existe dans la gelée un facteur inhibiteur de l'acide azélaïque (qui s'y trouve en très faible quantité) ou que la sécrétion de la glande mandibulaire lorsqu'elle se répand sur les téguments, y subit diverses modifications. 
TABL. 4. - Action sur le rejet de $\mathrm{CO}_{2}$ de têtes séparées du corps ou de thorax + abdomen sans la tête. Expériences réalisées entre le $1^{\text {er }}$ et le 20 février 1984. Les expériences sont présentées par couples du même jour. Dans chaque expérience 3 abeilles ont été l'objet de mesures répétées 3 fois.

TABL. 4. - Action upon $\mathrm{CO}_{2}$ elimination of heads of honeybees or thorax + abdomen without head. Tests performed from $1 / 2-20 / 2 / 1984$. In each experiment three honeybees were tested three times the same day.

\begin{tabular}{|c|c|c|c|}
\hline Thorax + abdomen & $T$ & $\begin{array}{l}\text { Tête isolée } \% \\
\text { Isolated head } * * *\end{array}$ & $\mathbf{T}$ \\
\hline 19 & 20 & 11 & 5 \\
\hline 29 & 25 & 19 & 29 \\
\hline 36 & 125 & 22 & 50 \\
\hline 15 & 20 & 30 & 12 \\
\hline 55 & 15 & 15 & 22 \\
\hline 10 & 28 & 12 & 29 \\
\hline 20 & 20 & 20 & 5 \\
\hline 15 & 20 & 15 & 29 \\
\hline 30 & 20 & 52 & 30 \\
\hline 15 & 10 & 10 & 10 \\
\hline 72 & 13 & 15 & 22 \\
\hline 12 & 5 & 15 & 12 \\
\hline 100 & 190 & 170 & 180 \\
\hline 165 & 150 & 10 & 190 \\
\hline 170 & 190 & 20 & 185 \\
\hline 60 & 20 & 90 & 55 \\
\hline 55 & 170 & 10 & 150 \\
\hline 45 & 150 & 15 & 80 \\
\hline 65 & 25 & 35 & 30 \\
\hline 35 & 65 & 10 & 17 \\
\hline 25 & 35 & 20 & 17 \\
\hline 170 & 40 & 26 & 20 \\
\hline 30 & 160 & 10 & 10 \\
\hline 37 & 30 & 20 & 20 \\
\hline 190 & 170 & 10 & 45 \\
\hline 105 & 190 & 120 & 15 \\
\hline 110 & 180 & 10 & 180 \\
\hline 120 & 10 & 135 & 35 \\
\hline 20 & 85 & 10 & 15 \\
\hline 20 & 25 & 85 & 190 \\
\hline 25 & 15 & 35 & 65 \\
\hline 20 & 25 & 17 & 18 \\
\hline
\end{tabular}


TABL. 4 (suite)

\begin{tabular}{|c|c|c|c|}
\hline Thorax + abdomen * & $T$ & $\begin{array}{l}\text { Tête isolée } * * \\
\text { Isolated head *** }\end{array}$ & $\mathbf{T}$ \\
\hline 10 & 140 & 26 & 60 \\
\hline 35 & 10 & 40 & 65 \\
\hline 20 & 25 & 12 & 20 \\
\hline 40 & 10 & 30 & 20 \\
\hline 31 & 60 & 35 & 18 \\
\hline 30 & 15 & 50 & 190 \\
\hline 160 & 75 & 150 & 195 \\
\hline 10 & 21 & 50 & 20 \\
\hline 15 & 10 & 30 & 160 \\
\hline 70 & 40 & 80 & 145 \\
\hline 25 & 25 & 15 & 20 \\
\hline 70 & 10 & 55 & 85 \\
\hline 10 & 10 & 60 & 100 \\
\hline 10 & 49 & 10 & 10 \\
\hline 25 & 30 & 45 & 15 \\
\hline 35 & 60 & 20 & 28 \\
\hline
\end{tabular}

* Student and Mann \& Whitney tests negative.

*** Student test positive at less than $1 \%$.

**: $*$ Fischer Snedecor test positive at $1 \%$.

\section{DISCUSSION}

En quoi consiste le stress de l'isolement?

Il semble qu'on doive placer l'accélération du métabolisme au premier rang : elle entraînerait une consommation accrue des lipides, et il n'est pas impossible que la déplétion en lipides gêne la formation des œufs ; d'autre part on n'ignore pas la part de la pars intercerebralis dans l'ovogenèse par l'intermédiaire des corpora allata. La diminution de la neurosécrétion de la pars intercerebralis est en corrélation avec l'accélération du métabolisme, mais quel est le phénomène premier? L'analyse du phénomène n'a pas été poussée assez loin pour qu'on puisse le dire. 


\title{
Qu'est-ce qui déclenche le stress de l'isolement?
}

Nous conclurons que c'est l'absence d'une substance «tranquilisante 》 en quelque sorte, puisqu'elle ralentit beaucoup le métabolisme : et il faut rappeler l'observation d'ARNOLD suivant lequel les abeilles isolées s'agitent beaucoup plus que les groupées. Les diacides, répandus sur le corps des abeilles (et non obtenus par échange de nourriture comme je le croyais en 1952) prolongent donc la vie de l'isolée. Il ne peut s'agir que d'une chimioréception de contact et l'abeille ne peut percevoir la substance en question sur son propre corps, soit que l'isolement en fasse cesser la sécrétion, soit qu'elle doive être associée à un corps étranger, rugueux ou pileux, bref à un stimulus tactile.

\section{Le problème des sécrétions tarsales de Lensky}

Cet auteur m'a communiqué les extraits des traces des ouvrières laissées sur le verre, et je les ai trouvés actifs sur la survie (Chauvin, 1981). Or, leur composition est tout à fait différente de celle de la survivone ; à peine ont-ils en commun quelques alcanes. Parmi ces constituants, l’acide décanoïque s'est révélé seul actif sur la survie et le métabolisme. J'avais émis dans un travail précédent l'idée que la survivone devait provenir de la grosse glande d'Anhart qui se trouve dans les tarses. Comme on le voit, je suis forcé de changer d'opinion.

Reçu pour publication en mars 1984. Accepté pour publication en septembre 1984.

\section{ZUSAMMENFASSUNG}

\author{
DIE WIRKUNG DES HORMONS «SURVIVONE» AUF DIE MORTALITAT \\ VON ISOLIERTEN HONIGBIENEN, APIS MELLIFERA L. (GRUPPENEFFEKT)
}

Atherextrakte von Arbeiterinnen der Honigbiene enthalten eine Substanz oder eine Gruppe von Substanzen, die das Leben von isolierten Bienen nachweislich verlängern (Tab. 1). Die Analyse des Extraktes im Gaschromatographen und durch Massenspektographie (nach Einwirkung von Wasserdampf) ergab folgende Inhaltsstoffe: Alkane, höhere Alkohole und vor allem eine Gruppe von Di- und Monosäuren. Der Einfluß dieser Stoffe auf das Überleben isolierter Bienen wurde mit einer einheitlichen Dosierung von $1 \mu \mathrm{g}$ getestet. Bis jetzt haben sich die Azelainsäure oder Nonandisäure und die Pimelinsäure oder Heptandisäure als wirksam herausgestellt (Tab. 2). Danach wurde die Wirkungsweise dieser Substanzen analysiert. Die respirometrischen Tests haben gezeigt, daß diese Säuren die Eigenschaft haben, die Produktion von $\mathrm{CO}_{2}$ durch die isolierten Bienen zu reduzieren (Tab. 3). Mit diesem respirometrischen Test wurde dann die Suche nach dem Produktionsort dieser Säuren aufgenommen. Man präsentierte den isolierten Bienen den Kopf, den Thorax oder das Abdomen von Stockgenossen. Man stellte fest, daß der Kopf bei weitem der wirksamste Körperteil ist (Tab. 4). Die Droge wirkt nicht durch Aufnahme in den Verdauungstrakt sondern durch Chemorezeption beim Kontakt. Wir wissen noch nicht, ob sie durch die Mandibeldṛüsen oder die Pharynxdrüsen gebildet wird. Jedenfalls findet man Azelainsäure im Gelée royale 


\title{
SUMMARY
}

\author{
ACTION OF A HORMONE, SURVIVONE, UPON ISOLATED HONEYBEE \\ (APIS MELLIFERA L.) MORTALITY (GROUP EFFECT)
}

Ether extracts of honeybee workers contain a substance, or a group of substances, that greatly extends the survival of isolated honeybees (Tabl. 1). The extracted product was steam distilled, then analysed by gas chromatography and mass spectrometry. Alcanes, higher alcohols and particularly a group of diacids and a few monacids were isolated. The action of these substances was tested on the survival of isolated honeybees at a dose of $1 \mu \mathrm{g}$. Until now only azelaic (or nonandioic) and pimelic (or heptandioic) acids were found to be efficient (Tabl. 2).

We attempted to determine the mode of action of these substances. Respirometric tests showed that these acids strongly reduced the production of carbon dioxide in isolated bees (Tabl. 3). They also enabled tests for the emission site of these acids to be conducted. Honeybee heads, thoraces and abdomens were given to isolated bees; the head is by far the most active body part (Tabl. 4).

The substance did not act by ingestion but by contact chemoreception. We do not yet know whether it is secreted by mandibular or pharyngeal glands, but azelaic acid occurs in royal jelly.

\section{BIBLIOGRAPHIE}

Arnold G., 1976. - Contribution à l'étude du déterminisme de l'effet de groupe chez Apis mellifica. Thèse Doct. $3^{\mathrm{e}}$ cycle, Paris, $184 \mathrm{p}$.

Chauvin R., 1979. - Sur la toxicité pour les abeilles des extraits d'abeilles adultes. Congrès Union Internationale pour l'Etude des Insectes Sociaux, Lausanne, 127-131.

Grasse P.P., Chauvin R., 1944. - L'effet de groupe et la survie des neutres dans les sociétés animales. Rev. Scient., 7, 461-464.

LENSKY Y. - (Communication personnelle).

Pain J., 1961. - Sur la phérormone des reines d'abeilles et ses effets physiologiques. Thèse Doct. Etat, publ, in : Ann. Abeille, 4, 73-151.

Pain J., Barbier M., 1963. - Structure chimique et propriétés biologiques de quelques substances identifiées chez l'abeille. Insectes Soc., 129-142.

Punt A., 1950. - The respiration of insects. Physiol. Comp. Oecol., 2, 59-74.

Roтн M., 1965. - La production de chaleur chez Apis mellifica. Thèse Doct. Ingénieur, Paris, publ. in : Ann. Abeille, 8 (1), 5-77.

Sitbon G., 1971. - Corpora allata, neurosécrétion et effet de groupe chez l'abeille d'hiver. Insectes Soc., 18, 161-172. 